\title{
Optimizing Transmission of IoT Nodes in Dynamic Environments
}

\author{
Gilles Callebaut, Geoffrey Ottoy, Liesbet Van der Perre \\ KU Leuven, ESAT-DRAMCO, Ghent Technology Campus, Ghent, Belgium \\ gilles.callebaut@kuleuven.be, geoffrey.ottoy@kuleuven.be, liesbet.vanderperre@kuleuven.be
}

\begin{abstract}
Many IoT applications require long range yet low power connectivity in dynamic environments. We assess and optimize their energy efficiency in a Long Range Wide Area Network (LoRaWAN) network following a cross-layer approach. The analysis demonstrates that the channel variation may significantly impact the quality of the transmission and the energy consumption of the nodes. A proactive adjustment strategy of the Adaptive Data Rate settings allows for optimization of the transmit energy. In addition, we show how trade-offs between robustness, energy efficiency and throughput can be made.
\end{abstract}

Index Terms-IoT, Energy Efficiency, LPWAN, Crosslayer, LoRaWAN

\section{INTRODUCTION}

Many applications, such as remote environmental monitoring and logistics, rely on energy constrained IoT devices to get connected at long range. Dedicated Low Power Wide Area Network (LPWAN) technologies are being deployed to support these. In this paper, we consider the LoRaWAN technology [1]. Remote sensing applications can use this technology to transmit a limited number of relatively short packets each day, i.e. the uplink. To improve reliability, devices have the possibility to request a receive confirmation using downlink messages.

Another interesting feature, is the option to automatically adjust transmission parameters whenever wireless channel conditions change. The Adaptive Data Rate (ADR) mechanism, attempts to achieve a robust signal while also taking into account the energy expenditure. This is achieved by lowering the data rate when the channel conditions worsen, and by increasing the data rate when the conditions improves.

Briefly put, lowering (resp. increasing) the data rate, will increase (resp. reduce) the energy-per-bit, effectively tuning the Signal-to-Noise Ratio (SNR). In this article, we investigate how the ADR algorithm's behaviour can be tuned to a changing channel in order to achieve either robustness or energy efficiency.

\section{A. LoRaWAN need-to-know}

LoRaWAN modulation is based on a spectrum spreading technique called Chirp Spread Spectrum (CSS). Where Direct-Sequence Spread Spectrum (DSSS) encodes information with pseudorandom codes, CSS uses chirps. Chirps are sinusoidal signals with a monotonically decreasing or increasing frequency. The spreading factor $(S F)$ determines the duration of a LoRa symbol. Since, each symbol is composed of $2^{S F}$ chirps, the symbol duration is defined as

$$
T_{\text {sym }}=\frac{2^{\mathrm{SF}}}{\mathrm{BW}},
$$

where $(B W)$ is the frequency bandwidth. Typically the bandwidth is fixed to either $125 \mathrm{kHz}, 250 \mathrm{kHz}$ or $500 \mathrm{kHz}$, depending your region and frequency plan.

Instead of defining a specific data rate, LoRaWAN specifies the data rate as a combination of a spreading factor and bandwidth (see first two columns of Table I). ${ }^{1}$ One can see that DR3 uses SF9, while DR2 uses SF10. Hence, with DR3, one can send a double amount of data in the same time. Similarly, since the symbol time for DR3 is half the symbol time for DR2, one can see that the energy-per-bit will also be half, i.e., if the same transmit power is used. Equivalently, this also holds for the SNR.

While in some applications, the loss of some packets is allowed, LoRaWAN also provides downlink messages to acknowledge the packet reception. Hence, when packet loss is not permitted, receiving these downlink messages and re-transmitting lost packets, will also impact the energy required to successfully transmit a packet. When either energy or robustness is a key application feature, choosing the right combination of ADR and (un-)confirmed messages is paramount.

\section{B. ADR evaluation: related work}

In order to evaluate the ADR mechanism under varying conditions, we utilize the framework of Callebaut et al., which is also available open-source. This framework is chosen over others $[3,4,5,6]$ because of the support of energy monitoring and the high coverage of the LoRaWAN specification such as ADR and downlink messages. Furthermore, the energy monitoring includes the energy consumption of a real Internet of Things (IoT) node [7].

Recent studies have evaluated the ADR behaviour in terms of convergence speed and energy consumption

\footnotetext{
${ }^{1}$ The data rates show here, are specific to the EU868, EU433, CN780 and AS923 bands.
} 
for changing environments and increasing numbers of devices. For example, Li et al. show that ADR reacts slowly in situations with many devices or a significant channel variance. For determining the energy efficiency, however, the study only seems to take into account the transmit power. A comparison of the energy efficiency when whether or not using ADR is also lacking.

Other studies focus on how to improve the ADR algorithm [9, 10, 11]. The authors of [9] propose several improvements to the default ADR algorithm including hysteresis in order to avoid excessive amounts of downlink messages. While the packet error ratio has been determined experimentally, and in on specific scenario, the energy consumption is only calculated based on the transmit power set by the device. Hence, the energy efficiency does not take into account the energy required for receiving downlink messages, or when the end device is in sleep.

Peruzzo and Vangelista suggest further improvements to the former work [10]. Their main focus is the impact of the link distance on the ADR behaviour. It is unclear if the simulations take into account collisions and retransmissions.

A completely different approach is taken by Abdelfadeel et al. [11]. The authors propose a fair ADR algorithm to maintain a comparable packet extraction rate for each device when the number of devices (and consequently the number of collisions) rises.

In the next section, we will give more details about the simulation setup and we will highlight the most important aspects of the ADR algorithm as well as the evaluation criteria. Sect. III gives an overview of the results and shows how both ADR and downlink messages impact the robustness, throughput and energy efficiency. We end this article with an overview of the conclusions and some suggestions for future work.

\section{Simulation FramewORK}

We discuss the ADR algorithm of the simulator and the simulation setup in more detail in this section.

\section{A. Adaptive Data Rate Algorithm}

As per recommendation [12], the algorithm uses the maximum SNR of the last 20 received uplink messages to determine the quality of the link between the node and the gateway. If the SNR is lower than required, the network tries to decrease the Data Rate or increase the transmit power. This mechanism is based on the SNR margin and is expressed as:

$$
\mathrm{SNR}_{\text {margin }}=\mathrm{SNR}_{\text {packets }}-\mathrm{SNR}_{\text {req }}(\mathrm{DR})-\text { margin . }
$$

$\mathrm{SNR}_{\text {packets }}$ depends on the choice of the SNR estimator. By default, the maximum of the SNR is taken, and is further denoted by $\mathrm{SNR}_{\max }$. The margin is the link margin and is by default $10 \mathrm{~dB}$. Increasing the link margin results in devices going to lower data rates, while decreasing the margin will force devices to increase their data rate. A minimal SNR is required to demodulate a LoRaWAN signal. The $\mathrm{SNR}_{\text {req }}(\mathrm{DR})$ function returns the demodulation floor with respect to the current Data Rate (DR). The demodulation floor for each DR is shown in Table I.

The $\mathrm{SNR}_{\text {margin }}$ is used to determine how to adapt to the link. The number of steps is computed as NStep = $\operatorname{round}\left(\mathrm{SNR}_{\text {margin }} / 3\right)$. In the case NStep is positive, the Data Rate can be increased and/or the power reduced. However, if NStep is negative, the transmit power is increased to accommodate for the link loss. This mechanism is illustrated in Figure 1. Note that the algorithm only increases the Data Rate. Lowering the DR could result in constantly oscillating values. As a result, the network only decreases the transmit power of the device, while the devices uses an automatic data rate decay when it detects it does not receive any response back from the network. This is of course only valid when ADR is enabled.

TABLE I: SNR Demodulation floor per data rate/spreading factor.

\begin{tabular}{lll}
\hline Data Rate & & SNR $_{\text {req }}(\mathrm{DR}) \mathrm{dB}$ \\
\hline DR5 & SF7BW125 & -7.5 \\
DR4 & SF8BW125 & -10 \\
DR3 & SF9BW125 & -12.5 \\
DR2 & SF10BW125 & -15 \\
DR1 & SF11BW125 & -17.5 \\
DR0 & SF12BW125 & -20 \\
\hline
\end{tabular}

\section{B. Simulation Setup}

In our experiments we have used a cross-layer framework to analyze and optimize the transmission of a set number of nodes [2] taking into account collisions in the network. Our goal is to study the effect of the channel dynamics and payload size on the effective throughput, energy efficiency, and collision ratio. The simulator uses a log-distance channel model with shadowing. This model characterizes the path loss as

$$
P L(d)=P L\left(d_{0}\right)+10 \cdot n \log \frac{d}{d_{0}}+X_{\sigma}[\mathrm{dB}]
$$

with $X_{\sigma} \sim \mathcal{N}\left(0, \sigma^{2}\right)$.

The default parameters used for our experiments is displayed in Table II. The network is evaluated by repeating the Monto-Carlo simulations 1000 times for each experiment. The nodes are randomly spread over the considered area. These nodes respect the imposed duty cycle limitations. In addition, the devices select a free channel with the lowest back-off time ${ }^{2}$ and waits till

\footnotetext{
${ }^{2}$ The back-off time depends on the duty cycle imposed at the operating frequency and the occupied air time, and is defined as (time-on-air/duty-cycle) - time-on-air.
} 


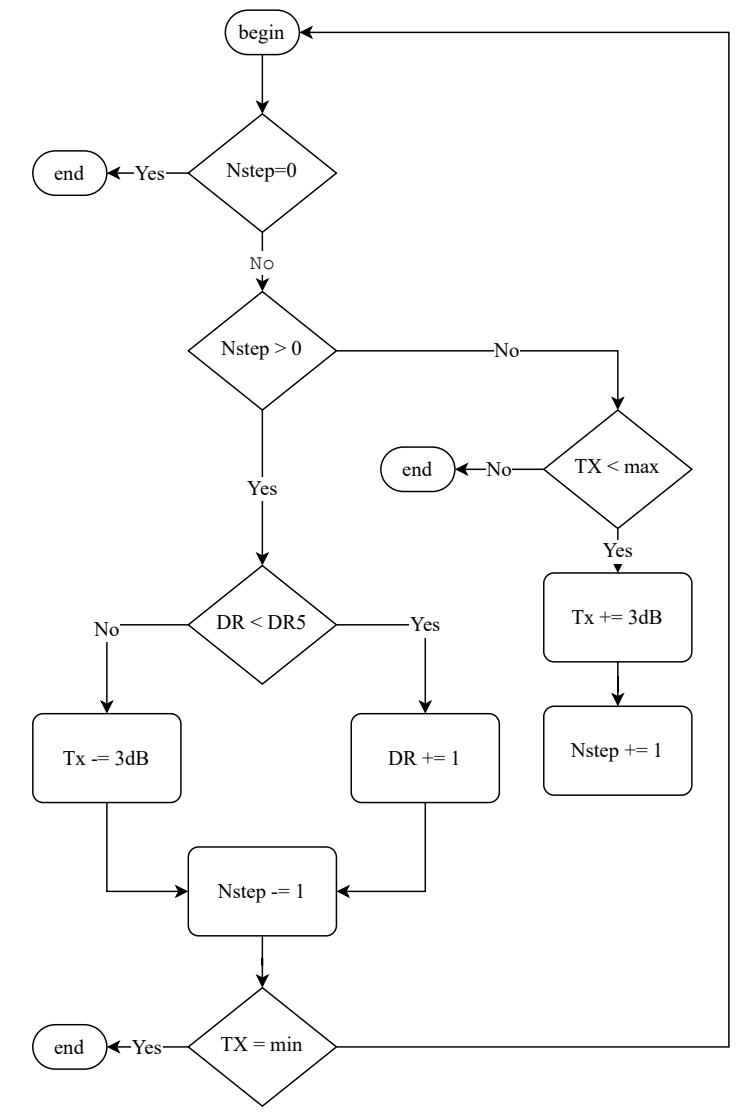

Fig. 1: Adaptive Data Rate mechanism to control the data rate and transmit power of nodes.

the back-off is zero before starting to transmit the (potentially queued) packets. A default transmission rate $(\lambda)$ of 0.02 bits per second is chosen which is equivalent to transmitting a message with a payload of 9 bytes every hour. All nodes have an initial transmit power of $14 \mathrm{dBm}$ conform the LoRaWAN specification. Only the channels required by the LoRaWAN specification are considered, i.e., $868.1 \mathrm{MHz}, 868.3 \mathrm{MHz}$ and $868.5 \mathrm{MHz}$. Semtech defines a spreading factor of 12 in the second receive window. However, in this work, we favor a spreading factor of 9 as proposed by The Things Network for two reasons: (I) a lower spreading factor results in a faster reception, which in turn yields a lower energy consumption of the node and (II) the LoRaWAN gateway utilizes a downlink channel allowing for a higher duty cycle and higher transmit power operation, so that loss due to lowering the spreading factor can be resolved by transmitting at a higher power.

\section{ENERGY EFFICIENCY AND THROUGHPUT ASSESSMENT AND OPTIMIZATION}

The channel dynamics are modeled through the variation of the standard deviation of the shadowing factor $\sigma$ in Eq. 3. The effect of the channel dynamics on the performance of both the network and individual nodes is assessed on the basis of the three parameters: the Data Extraction Rate, the energy per successfully received payload byte $\left(E_{B}\right)$ and the collisions ratio. These parameters are further defined in Sect. III-A. Sections III-B and III-C discuss the impact of different network parameters on the aforementioned evaluation criteria.

\section{A. Evaluation Criteria}

Data Extraction Rate. The Data Extraction Rate (DER) indicates how reliable the intended payload bytes are received by the gateway. It differs from the packet success rate because DER only takes into account the amount of uniquely transmitted and received packets, while the packet success rate considers all transmitted packets. The DER defines the average ratio of the number of uniquely received packets on the base station to the uniquely transmitted packets per node.

$$
D E R=\frac{1}{N} \frac{1}{J} \sum_{j}^{J} \sum_{n}^{N} \frac{U_{r x, n, j}}{U_{t x, n, j}}
$$

Note that the DER can be improved by utilizing retransmissions in order to accommodate for packet loss. A more elaborate description of these parameters can be found in [2].

Energy Efficiency $\left(E_{B}\right)$. The ratio of the average (total) energy consumed per node with respect to the number of successfully and uniquely received payload bytes per node is expressed as:

$$
E_{B}=\frac{1}{N} \frac{1}{J} \sum_{j}^{J} \sum_{n}^{N} \frac{E_{n, j}}{U_{r x, n, j}} .
$$

In the experiments, it illustrates the impact of different network parameters on the energy efficiency.

Collision Ratio (\%). The collision ratio is defined as the number of collided packets with respect to the total number of unique bytes transmitted:

$$
\frac{1}{N} \frac{1}{J} \sum_{j}^{J} C_{j} \sum_{n}^{N} \frac{1}{U_{r x, n, j}} \cdot 100(\%) .
$$

TABLE II: Parameters for the experiments reported on.

\begin{tabular}{ll}
\hline Parameter & Default Value \\
\hline Channel Variance $\sigma$ & $7.8 \mathrm{~dB}$ \\
Number of Nodes & 100 \\
Data Transmission rate $\lambda$ & $0.02 \mathrm{bps}$ \\
Initial Transmit Power & $14 \mathrm{dBm}$ \\
Channels & $868.1,868.3$ and $868.5 \mathrm{MHz}$ \\
RX2 Channel & $868.525 \mathrm{MHz}$ \\
RX2 Data Rate & DR3 (SF 9) \\
Cell Radius & $1000 \mathrm{~m}$ \\
Simulated time & $24 \mathrm{~h}$ \\
\hline
\end{tabular}




\begin{tabular}{|c|c|}
\hline Symbol & Description \\
\hline$N$ & Number of nodes. \\
\hline$J$ & Number of Monto-Carlo Simulations. \\
\hline$E_{n, j}$ & $\begin{array}{l}\text { Total energy consumed by node } n \text { in Monto-Carlo simu- } \\
\text { lation } j \text {. }\end{array}$ \\
\hline$B_{r x, n}$ & $\begin{array}{l}\text { Total number of bytes received by the gateway from node } \\
n \text {, including retransmissions. }\end{array}$ \\
\hline$B_{t x, n}$ & $\begin{array}{l}\text { Total number of bytes transmitted by the gateway from } \\
\text { node } n \text {, including retransmissions. }\end{array}$ \\
\hline$U_{r x, n}$ & $\begin{array}{l}\text { Uniquely number of bytes received by the gateway from } \\
\text { node } n \text {, i.e., excluding retransmissions. }\end{array}$ \\
\hline$U_{t x, n}$ & $\begin{array}{l}\text { Uniquely number of bytes received by the gateway from } \\
\text { node } n \text {, i.e., excluding retransmissions. }\end{array}$ \\
\hline$C_{j}$ & $\begin{array}{l}\text { Number of collided packets registered by the simulator } \\
\text { in Monto-Carlo simulation } j \text {. }\end{array}$ \\
\hline $\mathrm{SNR}_{\max }$ & $\begin{array}{l}\text { SNR estimator using the maximum of the SNR of the } \\
\text { packets, used in the default ADR algorithm. }\end{array}$ \\
\hline $\mathrm{SNR}_{\min }$ & $\begin{array}{l}\text { SNR estimator using the minimum of the SNR of the } \\
\text { packets, proposed as a potential condidate for the ADR } \\
\text { algorithm. }\end{array}$ \\
\hline
\end{tabular}

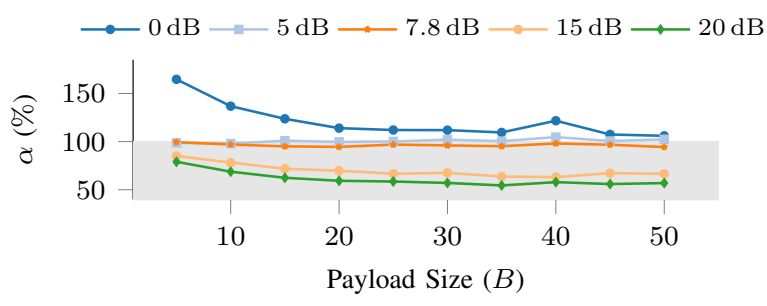

(a) The maximum SNR criteria is favored when the channel is more varying in terms of energy efficiency.

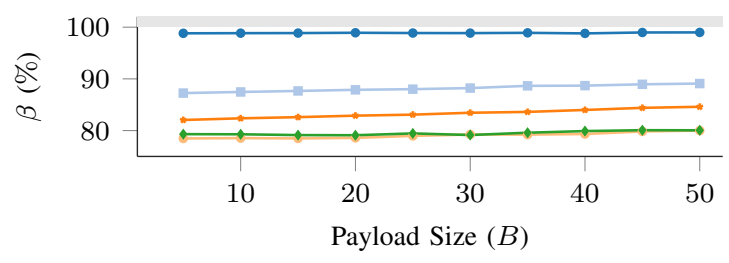

(b) A minimum SNR criteria always improves the DER.

Fig. 2: The grey area indicates when the maximum (default) SNR criteria is preferred rather than the minimum SNR for the energy (Eq. 7) and data extraction rate (Eq. 8), respectively.

SNR Estimator. The default implementation of ADR at the network side considers the maximum SNR of the uplink packets to determine how to adapt to the link. In that case, the reliability of the link is overestimated. In contrast, if the ADR mechanism would employ a minimum SNR criterion, the link would be underestimated. The influence of either selection criterion is evaluated based on both DER and $E_{B}$ and parameterized as:

$$
\begin{aligned}
\alpha & =\frac{E_{B, \mathrm{SNR}_{\text {max }}}}{E_{B, \mathrm{SNR}_{\text {min }}}} \\
\beta & =\frac{\mathrm{DER}_{\mathrm{SNR}_{\text {max }}}}{\mathrm{DER}_{\mathrm{SNR}_{\text {min }}}} .
\end{aligned}
$$

If $\alpha$ is higher than $100 \%$ and $\beta$ is lower than $100 \%$ both the $E_{b}$ and $D E R$ are improved when utilizing the minimum SNR selection criterion. The SNR selection criteria are denoted by $\mathrm{SNR}_{\max }$ and $\mathrm{SNR}_{\min }$. Note, that The Things Network employs the $\mathrm{SNR}_{\max }$ as an SNR estimator as recommended in [12].

\section{B. Impact of enabling $A D R$ and confirmation messages}

ADR reduces the energy consumption of the nodes. First, it optimizes the LoRa parameters such that nodes do not transmit with more power than needed. Secondly, this inherently diverges the utilized spreading factors yielding fewer collisions. The number of collisions is further reduced when not requesting downlink data, as depicted in Figure 3.

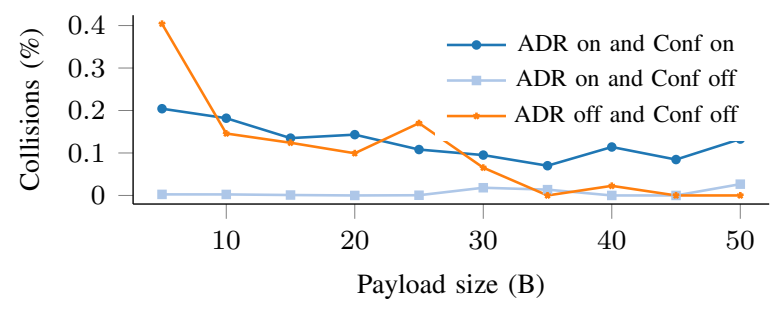

Fig. 3: Collisions ratio (w.r.t. the total bytes sent) for different configurations of ADR and confirmed messages.

However, the current ADR mechanism is unable to adjust for the rapid channel variances, as shown in Figure 4. This effect is negligible if confirmed messages (i.e., re-transmissions) are employed to ensure receiving each intended message. If no acknowledgments are used, the DER decreases when the channel variance increases. The nodes are unable to adequately adapt to the channel. With respect to the DER, it is even better to not enable adaptive data rate when not using confirmed messages. However, this results in a hefty energy penalty.

\section{Impact of the SNR Estimator}

Two SNR estimators are considered in this experiment: $\mathrm{SNR}_{\max }$ and $\mathrm{SNR}_{\min }$. The former is the default estimator which uses the maximum SNR of the received packets. The latter is an estimator which underestimates the channel conditions by taking the minimum of the SNR. These estimators are evaluated by $\alpha$ (Eq. 7) and $\beta$ (Eq. 8), considering the energy and Data Extraction Rate efficiency respectively. Figures $2 \mathrm{a}$ and $2 \mathrm{~b}$ demonstrate when $\mathrm{SNR}_{\max }$ is favored over $\mathrm{SNR}_{\text {min }}$, i.e., $\alpha<100 \%$ or $\beta>100 \%$, for different payload size under different channel conditions.

Impact on the DER due to the SNR Estimator. The $\mathrm{SNR}_{\text {min }}$ estimator always results in an DER improvement. This is obvious, considering that the path loss is always being overestimated. In addition, the payload size has only a negligible effect on the DER ratio. Even in the case of a variance of $7.8 \mathrm{~dB}$ (as measured in [13]), the 
Energy per Byte $(\mathrm{mJ})$
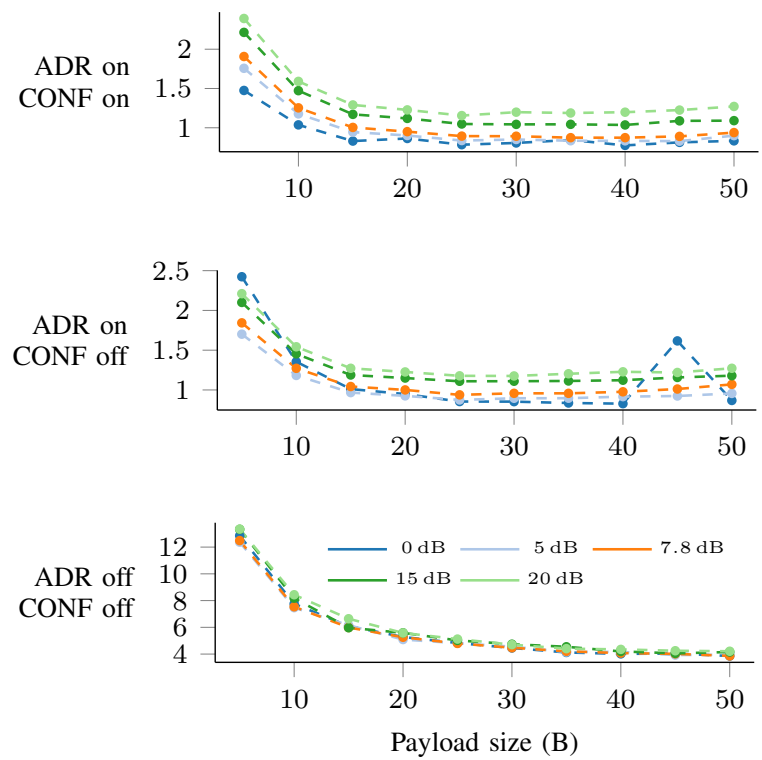

Data Extraction Rate (\%)
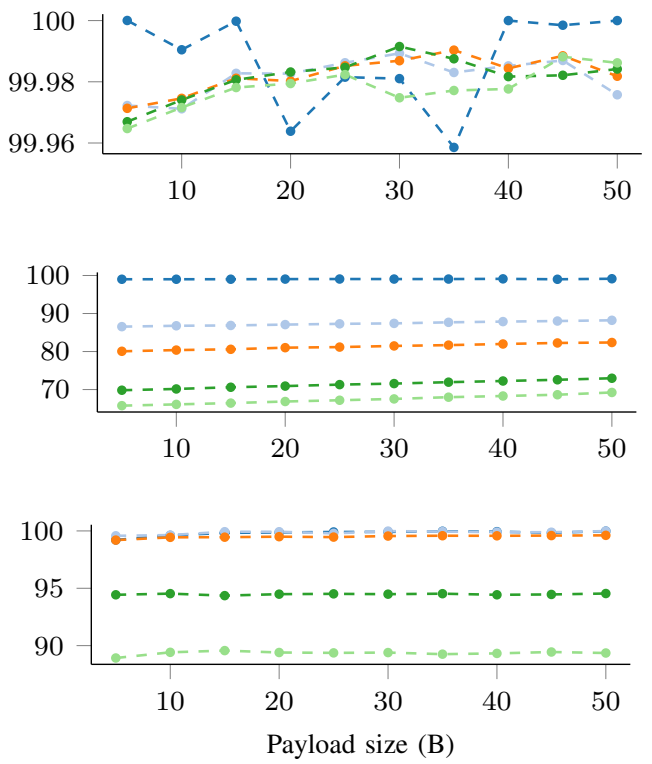

Fig. 4: Impact of the channel variance and payload size on the energy and data extraction rate. (based on [2])

DER can be improved without significantly decreasing the energy efficiency.

Impact on the energy efficiency due to the SNR Estimator. In contrast to the DER case, $\mathrm{SNR}_{\max }$ is more resilient against varying link conditions with respect to the energy efficiency. Moreover, $\alpha$ decreases by increasing the payload for high channel variance. In contrast, the $\mathrm{SNR}_{\text {min }}$ estimator is favored when the channel is static.

\section{CONCLUSiOnS AND FUture Work}

Channel dynamics have a significant impact on the performance of a LoRaWAN network and the energy efficiency of the nodes. The configuration of LoRaWANspecific parameters can anticipate on variations. The network-related Adaptive Data Rate mechanism can be exploited to optimize the energy and data extraction rate taking into account the channel variance. Utilizing different SNR selection criteria allows to make a tradeoff between energy efficiency and DER. Furthemore, we show the impact of using the ADR mechanism and (un-)confirmed messages on the energy efficiency and effective throughput. Future work will experimentally investigate both the channel variations and the strategies for optimizing energy efficient connectivity of IoT nodes connected in a LoRaWAN network. The impact of deploying large numbers of nodes is another track that is worth investigating.

\section{REFERENCES}

[1] N. Sornin and A. Yegin, LoRaWAN ${ }^{\mathrm{TM}}$ Specification, Std., 2017, v1.1.
[2] G. Callebaut, G. Ottoy, and L. van der Perre, "Cross-layer framework and optimization for efficient use of the energy budget of iot nodes," in 2019 IEEE Wireless Communications and Networking Conference (WCNC), 2019, pp. 1-6.

[3] M. Bor and U. Roedig, "Lora transmission parameter selection," in Proceedings of the 13th IEEE International Conference on Distributed Computing in Sensor Systems (DCOSS), Ottawa, ON, Canada, 2017, pp. 5-7.

[4] M. Slabicki, G. Premsankar, and M. Di Francesco, "Adaptive configuration of lora networks for dense iot deployments," 2018.

[5] B. Reynders, Q. Wang, P. Tuset-Peiro, X. Vilajosana, and S. Pollin, "Improving reliability and scalability of lorawans through lightweight scheduling," IEEE Internet of Things Journal, vol. PP, no. 99, pp. 1-1, 2018.

[6] A.-I. Pop, U. Raza, P. Kulkarni, and M. Sooriyabandara, "Does bidirectional traffic do more harm than good in lorawan based lpwa networks?" arXiv preprint arXiv:1704.04174, 2017.

[7] G. Ottoy, G. Leenders, and G. Callebaut, "LoRaWAN EFM32," doi: 10.5281/zenodo.1209414. [Online]. Available: https://github.com/DRAMCO/LoRaWAN_EFM32

[8] S. Li, U. Raza, and A. Khan, "How agile is the adaptive data rate mechanism of lorawan?" 08 2018, preprint.

[9] V. Hauser and T. Hégr, "Proposal of adaptive data rate algorithm for lorawan-based infrastructure," in 2017 IEEE 5th International Conference on Future Internet of Things and Cloud (FiCloud), 2017, pp. 85-90.

[10] A. Peruzzo and L. Vangelista, "A power efficient adaptive data rate algorithm for lorawan networks," in 201821 st International Symposium on Wireless Personal Multimedia Communications (WPMC), 2018, pp. 90-94.

[11] K. Q. Abdelfadeel, V. Cionca, and D. Pesch, "A fair adaptive data rate algorithm for lorawan," CoRR, vol. abs/1801.00522, 2018. [Online]. Available: http://arxiv.org/abs/1801.00522

[12] Semtech, "LoRaWAN - simple rate adaptation recommended algorithm," Tech. Rep.

[13] J. Petajajarvi, K. Mikhaylov, A. Roivainen, T. Hanninen, and M. Pettissalo, "On the coverage of LPWANs: range evaluation and channel attenuation model for LoRa technology," in ITS Telecommunications (ITST), 2015 14th International Conference on. IEEE, 2015, pp. 55-59. 